\title{
GLOBAL EXISTENCE AND EXPONENTIAL DECAY TO THE WAVE EQUATION WITH LOCALIZED FRICTIONAL DAMPING*
}

\author{
Yolanda S. Santiago Ayala
}

\author{
Jaime E. Muñoz Rivera**
}

ABSTRACT.- In this paper we show the global existence and the exponencial decay of solutions of the one-dimensional wave equation with localized frictional damping.

\section{INTRODUCTION}

In this paper we study the existence of global smooth solution to the wave equations when the dissipation given by the frictional term is effective only in a part of the domain. Moreover we show that this local dissipation is strong enough to produce uniform decay of the solutions. Let us consider a string o length $L$. The mathematical model which defines the deformations of the string is given by

$$
\left.u_{t t}-\left[\sigma\left(u_{x}\right)\right]_{x}+a(x) u_{t}=0 \text { in }\right] 0, L[x] 0, \infty[
$$

with initial data

$$
\left.u(x, 0)=u_{0}(x) \quad u_{t}(x, 0)=u_{1}(x) \text { in }\right] 0, L[
$$

and boundary conditions

$$
u(L, t)=u(0, t)=0 \quad \forall t \geq 0
$$

where by $u=u(x, t)$ we denote the displacement, $\sigma$ is a function satisfying

$$
\begin{aligned}
& \sigma \in C^{3}(\mathbb{R}) \text { such that } \sigma^{\prime}(0)>0 \text { and } \\
& \left.\left.a \in C^{1}(0, L) \quad a \geq 0, \quad a(x)>0 \quad \text { in }\right] L_{0}, L\right] \text { where } 0 \leq L_{0}<L .
\end{aligned}
$$

It is well known by now that in non linear elasticity when $a \equiv 0$, the equation is conservative and the solution will blow up in a finite time, no matter how small and regular the initial data is. On the other hand, when the dissipation is effective in the whole domain, then when the initial data is taken small enough there exists only one global smooth solution of the system.

\footnotetext{
* Supported by a grant $142520 / 96$ - 9 of CNPq (Brasil)

** Laboratorio Nacional de Computação Científica - LNCC - BRASIL
} 
The problem we consider here is intermediary between this two cases. The natural questions under in our case is about the strongness of the dissipation. We can ask, is the local dissipation strong enough to secure estimates and prevent blow up?. The mains result of this paper is to give a positive answer to that question. We will prove that the problem (1.1) - (1.5) is well posed, that is if $a \geq 0$, with $a$ zero outside of a boundy of 0 or $L$, the problem has a global smooth solution which decays exponentially as time goes to infinity, provided the initial data is small enough.

The main result of this paper is summarized in the following Theorem.

Theorem 1.1 (Main Result) Let us suppose that the initial data satisfies

$$
\begin{aligned}
& u_{0} \in H^{3}(0, L) \cap H_{0}^{1}(0, L), \quad u_{1} \in H^{2}(0, L) \cap H_{0}^{1}(0, L) \quad \text { such that } \\
& \left\|u_{0}\right\|_{H^{3}(0, L)}^{2}+\left\|u_{1}\right\|_{H^{2}(0, L)}^{2}+\left\|u_{t t}(0)\right\|_{H^{1}(0, L)}^{2}<\varepsilon^{2}
\end{aligned}
$$

for any $\varepsilon>0$ and small enough, where $u_{t t}(0)=\sigma^{\prime}\left(u_{0, x}\right) u_{0, x x}-a(x) u_{1}$, the there exists a global smooth solution of problem (1.1)- (1.5) satisfying $u \in C\left(0, \infty ; H^{3}\right) \cap C^{1}\left(0, \infty ; H^{2}\right) \cap C^{2}\left(0, \infty ; H^{1}\right)$, which decays exponentially as time goes to infinity.

The method we use is based on the multiplicative technique. We introduce a new multiplicator which allows us to get the global estimate that we need to show the global existence result.

The main ideia is to construct a Liapunov functional $\mathcal{L}$ satisfying

$$
\frac{d}{d t} \mathcal{L}(t) \leq-\mathcal{K} \mathcal{L}(t)
$$

where $\mathcal{K}$ is a positive constant.

\section{EXISTENCE AND ASYMPTOTIC BEHAVIOUR}

Our first step is to stablish the local existence result.

Theorem 2.1 (Local Existence) Let us take initial data satisfying

$$
u_{0} \in H^{3}(0, L) \cap H_{0}^{1}(0, L), \quad u_{1} \in H^{2}(0, L) \cap H_{0}^{1}(0, L)
$$

then, there exists $T>0$ and a function

$$
u \in X:=C\left(0, T ; \dot{H}^{3}\right) \cap C^{1}\left(0, T ; H^{2}\right) \cap C^{2}\left(0, T ; H^{1}\right)
$$

satisfying problem (1.1) - (1.5). 
Proof.- The main idea is to use the fixed point Theorem. To do this we define

$$
\begin{gathered}
W_{\mu}(0, T):=\left\{w \in C^{0}\left(0, T ; H^{2}\right), w \in C^{i}\left(0, T ; H^{3-i}\right) i=1,2\right. \\
\left.w(x, 0)=u_{0}(x), w_{t}(x, 0)=u_{1}(x),\|w\|_{W} \leq \mu\right\} \\
T: W_{\mu} \rightarrow W_{\mu} \\
v \rightarrow T v=u
\end{gathered}
$$

where $u$ is the solution of

$$
\left\{\begin{array}{l}
u_{t t}-\sigma^{\prime}\left(v_{x}\right) u_{x x}+a(x) u_{t}=0 \\
u(0)=u_{0} \in H^{2}(0, L), \quad u_{t}(0)=u_{1} \in H^{1}(0, L) \\
u(L, t)=u(0, t)=0
\end{array}\right.
$$

To show the global existence, it is enough to show that

$$
\lim _{t \rightarrow T_{\max }}\|u(t)\|_{X}<C
$$

Here we follows similar techniques as in [7]. Firts study the linearized problem.

$$
\left.u_{t t}-\sigma^{\prime}(0) u_{x x}+a(x) u_{t}=f \quad \text { in } \quad\right] 0, L[x] 0, T[
$$

where

$$
f=\left[\sigma^{\prime}\left(u_{x}\right)-\sigma^{\prime}(0)\right] u_{x x}
$$

In general we consider

$$
\begin{gathered}
\left.U_{t t}-\sigma^{\prime}(0) U_{x x}+a(x) U_{t}=F \quad \text { in }\right] 0, L[x] 0, T[ \\
U(L, t)=U(0, t)=0 \quad \forall t \geq 0
\end{gathered}
$$

where by $U, F$ we are denoting $(U, F)=\left\{(u, f),\left(u_{t}, f_{t}\right),\left(u_{t t}, f_{t t}\right)\right\}$.

Under the above notations the energy associated is

$$
E(t, U)=\frac{1}{2} \int_{0}^{L} U_{t}^{2}+\sigma^{\prime}(0) U_{x}^{2} d x
$$

Multiplying (2.2) by $U_{t}$, performing an integration by parts and using the boundary conditions we get

$$
\frac{d E(t, U)}{d t}=-\int_{0}^{L} a(x) U_{t}^{2} d x+\int_{0}^{L} F U_{t} d x
$$


Observation 1 The Lemas 2.1, 2.2, 2.3 that will be enunciates, are valid in the cases

$$
(U, F)=\left\{(u, f),\left(u_{t}, f_{t}\right),\left(u_{t t}, f_{t t}\right)\right\}
$$

Let us introduce the following functionals

$$
\begin{aligned}
& I(t, U)=\int_{0}^{L} \alpha(x) U U_{t} d x+\frac{1}{2} \int_{0}^{L} a(x) \alpha(x)|U|^{2}(x) d x \\
& J(t, U)=\int_{0}^{L} U_{t} q(x) U_{x} d x \\
& L(t, U)=N E(t, U)+I(t, U)+J(t, U)
\end{aligned}
$$

where

$$
\begin{aligned}
& \alpha \in C^{2}([0, L]), \quad \alpha(0)<0, \alpha(L)>0, \quad \alpha^{\prime \prime} \leq 0, \\
& q \in C^{1}(0, L), \quad q(0)=q(L)=0 \quad \text { and } \alpha, q \text { satisfying } \\
& \alpha(x)+\frac{1}{2} q^{\prime}(x) \geq C>0 \quad \text { and } \exists \alpha_{0}>0 \text { such that } \\
& \alpha_{0} a(x)-\alpha(x)+\frac{1}{2} q^{\prime}(x) \geq D>0
\end{aligned}
$$

The above conditions over $\alpha$ imply that there exists $L^{*} \in\left(0, L_{0}\right)$ such that $\alpha\left(L^{*}\right)=0$, therefore we have

$$
q^{\prime}(x):=\left\{\begin{array}{lll}
\alpha(x)+C & \text { in } & {\left[L^{*}, L_{0}+\delta_{1}\right]} \\
-\alpha(x)+C & \text { in } & {\left[0, L^{*}\right] \cup\left[L_{0}+\delta_{2}, L\right]}
\end{array}\right.
$$

such that $q^{\prime} \in C(0, L)$. Since $0<\delta_{1}<\delta_{2}$ taking $\int_{0}^{L} q^{\prime}(x) d x=0$.

Lema 2.1 Let $a, \sigma, \alpha$ be as (1.5), (1.4), (2.6) and $U$ be the strong solution of (2.2)(2.3) then the following inequalities follows

$$
\frac{d I(t, U)}{d t} \leq-\sigma^{\prime}(0) \int_{0}^{L} \alpha(x)\left|U_{x}\right|^{2} d x+\int_{0}^{L} \alpha(x)\left|U_{t}\right|^{2} d x+\int_{0}^{L} \alpha(x) U F d x
$$

Proof.- Multiplying (2.2) by $\alpha(x) U$ and integrating over $] 0, L[$, we find that

$$
\frac{1}{2} \frac{d}{d t} \int_{0}^{L} \alpha(x) a(x)|U|^{2} d x=\int_{0}^{L} F \alpha(x) U d x-\int_{0}^{L} \alpha(x) U U_{t t} d x+\sigma^{\prime}(0) \int_{0}^{L} \alpha(x) U U_{x x} d x
$$


Since

$$
\begin{aligned}
\int_{0}^{L} \alpha(x) U U_{x x} d x & =-\int_{0}^{L} \alpha^{\prime}(x) U U_{x} d x-\int_{0}^{L} \alpha(x)\left|U_{x}\right|^{2} d x \\
& =\frac{1}{2} \int_{0}^{L} \alpha^{\prime \prime}(x)|U|^{2} d x-\int_{0}^{L} \alpha(x)\left|U_{x}\right|^{2} d x
\end{aligned}
$$

Using (2.9) and $\alpha^{\prime \prime}(x) \leq 0,(2.8)$ will be

$$
\frac{1}{2} \frac{d}{d t} \int_{0}^{L} \alpha(x) a(x)|U|^{2} d x \leq \int_{0}^{L} F \alpha(x) U d x-\int_{0}^{L} \alpha(x) U U_{t t} d x-\sigma^{\prime}(0) \int_{0}^{L} \alpha(x)\left|U_{x}\right|^{2} d x
$$

Since

$$
\frac{d}{d t} \int_{0}^{L} \alpha(x) U U_{t} d x=\int_{0}^{L} \alpha(x)\left|U_{t}\right|^{2} d x+\int_{0}^{L} \alpha(x) U U_{t t} d x
$$

Summing up the two above relations our conclusion follows.

Lemma 2.2 Let $a, \sigma, \alpha$ be as in (1.5), (1.4) and $U$ be the strong solution of (2.2) (2.3) then the following inequality holds

$$
\begin{aligned}
\frac{d J(t, U)}{d t}= & \frac{\sigma^{\prime}(0)}{2} \underbrace{\left\{\left|U_{x}\right|^{2}(L, t) \varphi(L)-\left|U_{x}\right|^{2}(0, t) \varphi(0)\right\}}_{:=b(t)} \\
& -\frac{1}{2} \int_{0}^{L} \varphi^{\prime}(x)\left\{\left|U_{t}\right|^{2}+\sigma^{\prime}(0)\left|U_{x}\right|^{2}\right\} d x-\int_{0}^{L} a(x) U_{t} \varphi(x) U_{x} d x \\
& +\int_{0}^{L} F \varphi(x) U_{x} d x
\end{aligned}
$$

for any $\varphi \in C^{1}(0, L)$.

Proof.- Multiplying equation (2.2) by $\varphi(x) U_{x}$ and integrating over $] 0, L[$ we find

$$
\int_{0}^{L} U_{t t} \varphi(x) U_{x} d x=\sigma^{\prime}(0) \int_{0}^{L} U_{x x} \varphi(x) U_{x} d x-\int_{0}^{L} a(x) \varphi(x) U_{t} U_{x} d x+\int_{0}^{L} F \varphi(x) U_{x} d x
$$

integrating by parts

$$
=\frac{-\sigma^{\prime}(0)}{2} \int_{0}^{L} \varphi^{\prime}(x)\left|U_{x}\right|^{2} d x+\left.\frac{\sigma^{\prime}(0)}{2} \varphi(x)\left|U_{x}\right|^{2}\right|_{0} ^{L}-\int_{0}^{L} a(x) \varphi(x) U_{t} U_{x} d x+\int_{0}^{L} F \varphi(x) U_{x} d x
$$


But

$$
\int_{0}^{L} U_{t t} \varphi(x) U_{x} d x=\frac{d}{d t} \int_{0}^{L} U_{t} \varphi U_{x} d x-\int_{0}^{L} U_{t} \varphi(x) U_{x t} d x
$$

integrating by parts

$$
=\frac{d}{d t} \int_{0}^{L} U_{t} \varphi U_{x} d x+\frac{1}{2} \int_{0}^{L} \varphi^{\prime}(x)\left|U_{t}\right|^{2} d x
$$

From relations (2.11) and (2.12), our conclusion follows.

Lemma 2.3 Let $a, \sigma, \alpha, q$ be as in (1.5), (1.4), (2.6) and let us take $N$ large enough, then there exists $\hat{\delta}>0$ such that

$$
\frac{d L(t, U)}{d t} \leq-\hat{\delta} E(t, U)+\int_{0}^{L} F\left\{N U_{t}+\alpha(x) U+q(x) U_{x}\right\} d x
$$

Moreover, there exist positive constants $k_{1}, k_{2}$ such that

$$
k_{1} E(t, U) \leq L(t, U) \leq k_{2} E(t, U)
$$

Proof.- From (2.5), (2.7), (2.10) and since $q(0)=q(L)=0$. (that is $b(t)=0$ ) we have

$$
\begin{gathered}
\frac{d L(t, U)}{d t} \leq \\
\underbrace{-N \int_{0}^{L} a(x)\left|U_{t}\right|^{2} d x-\sigma^{\prime}(0) \int_{0}^{L} \alpha(x)\left|U_{x}\right|^{2} d x-\frac{1}{2} \int_{0}^{L} q^{\prime}(x)\left\{\left|U_{t}\right|^{2}+\sigma^{\prime}(0)\left|U_{x}\right|^{2}\right\} d x}_{:=\mathrm{I}_{12}} \\
+\underbrace{\int_{0}^{L} \alpha(x)\left|U_{t}\right|^{2} d x}_{:=\mathrm{I}_{11}}-\underbrace{-\int_{0}^{L} a(x) U_{t} q(x) U_{x} d x}_{0}+\int_{0}^{L} F\left\{N U_{t}+\alpha(x) U+q(x) U_{x}\right\} d x
\end{gathered}
$$

Let us find some estimates to $I_{1}, I_{2}$, where $I_{1}=I_{11}+I_{12}$

$$
\begin{aligned}
& I_{1}=-\int_{0}^{L}\left[N a(x)-\alpha(x)+\frac{1}{2} q^{\prime}(x)\right]\left|U_{t}\right|^{2} d x-\sigma^{\prime}(0) \int_{0}^{L}\left[\alpha(x)+\frac{1}{2} q^{\prime}(x)\right]\left|U_{x}\right|^{2} d x \\
& I_{2}=-\int_{0}^{L}\left(\frac{1}{\sqrt{2 \varepsilon}} \sqrt{a} U_{t}\right)\left(\sqrt{2 \varepsilon} \sqrt{a} q U_{x}\right) d x
\end{aligned}
$$


Summing up $I_{1}$ with $I_{2}$ we get

$$
\begin{aligned}
I_{1}+I_{2} \leq- & \int_{0}^{L}\left[\left(N-\frac{1}{4 \varepsilon}\right) a(x)-\alpha(x)+\frac{1}{2} q^{\prime}(x)\right]\left|U_{t}\right|^{2} d x- \\
& \sigma^{\prime}(0) \int_{0}^{L}\left[\alpha(x)+\frac{1}{2} q^{\prime}(x)\right]\left|U_{x}\right|^{2} d x+\varepsilon \underbrace{\int_{0}^{L} a q^{2}\left|U_{x}\right|^{2} d x}_{:=I_{3}} .
\end{aligned}
$$

Since,

$$
I_{3}=\int_{L_{0}}^{L} a(x) q^{2}\left|U_{x}\right|^{2} d x \leq \underbrace{\left(\max _{x \in\left[L_{0}, L\right]}|q(x)|^{2}\right)}_{:=\mathrm{M}_{0}} \int_{L_{0}}^{L}\left|U_{x}\right|^{2} d x
$$

we can write

$$
\begin{aligned}
I_{1}+I_{2} \leq & -\int_{0}^{L}\left[\left(N-\frac{1}{4 \varepsilon}\right) a(x)-\alpha(x)+\frac{1}{2} q^{\prime}(x)\right]\left|U_{t}\right|^{2} d x-\int_{L_{0}}^{L} \sigma^{\prime}(0)\left[\alpha(x)+\frac{1}{2} q^{\prime}(x)\right]\left|U_{x}\right|^{2} d x \\
& -\int_{0}^{L_{0}} \sigma^{\prime}(0)\left[\alpha(x)+\frac{1}{2} q^{\prime}(x)\right]\left|U_{x}\right|^{2} d x+\varepsilon M_{0} \int_{L_{0}}^{L}\left|U_{x}\right|^{2} d x
\end{aligned}
$$

where $\alpha$ and q must satisfy $\alpha(x)+\frac{1}{2} q^{\prime}(x) \geq C>0$ in $[0, L]$. Let us take, $\varepsilon$ such that $\varepsilon<\frac{C}{M_{0}} \sigma^{\prime}(0)$ and $N$ such that

$$
N>\frac{1}{4 \varepsilon} \text { and }\left[\left(N-\frac{1}{4 \varepsilon}\right) a(x)-\alpha(x)+\frac{1}{2} q^{\prime}(x)\right] \geq D>0 \text {. }
$$

where,

$$
\begin{aligned}
& C=\min _{x \in[0, L]}\left\{\alpha(x)+\frac{1}{2} q^{\prime}(x)\right\} \\
& D=\min _{x \in[0, L]}\left\{\left(N-\frac{1}{4 \varepsilon}\right) a(x)-\alpha(x)+\frac{1}{2} q^{\prime}(x)\right\}
\end{aligned}
$$

Under the above notations we can rewrite inequality (2.16) in the following way 


$$
\begin{aligned}
I_{1}+I_{2} & \leq-D \int_{0}^{L}\left|U_{t}\right|^{2} d x-\left(\sigma^{\prime}(0) C-\varepsilon M_{0}\right) \int_{L_{0}}^{L}\left|U_{x}\right|^{2} d x-\sigma^{\prime}(0) C \int_{0}^{L_{0}}\left|U_{x}\right|^{2} d x \\
& \leq-D \int_{0}^{L}\left|U_{t}\right|^{2} d x-\left(\sigma^{\prime}(0) C-\varepsilon M_{0}\right) \int_{L_{0}}^{L}\left|U_{x}\right|^{2} d x \\
& \leq-2 \min \left\{D, C-\frac{\varepsilon M_{0}}{\sigma^{\prime}(0)}\right\} \frac{1}{2} \int_{0}^{L}\left|U_{t}\right|^{2}+\sigma^{\prime}(0)\left|U_{x}\right|^{2} d x \\
& \leq-\underbrace{2 \min \left\{D, C-\frac{\varepsilon M_{0}}{\sigma^{\prime}(0)}\right\} E(t, U)}_{:=\hat{\delta}}
\end{aligned}
$$

We finish the proof of (2.13) using relation (2.17) in (2.15). To show (2.14), we proced as follows

$$
\begin{aligned}
\left|\int_{0}^{L} \alpha(x) U U_{t} d x\right| & \leq|\alpha|_{\infty} \frac{1}{2} \int_{0}^{L}|U|^{2}+\left|U_{t}\right|^{2} d x \\
\left.\left|\int_{0}^{L} a(x) \alpha(x)\right| U\right|^{2}(x) d x \mid & \leq|\alpha|_{\infty} \frac{1}{2} \int_{0}^{L}|U|^{2} d x \\
\left|\int_{0}^{L} q(x) U_{x} U_{t} d x\right| & \leq|q|_{\infty} \frac{1}{2} \int_{0}^{L}\left|U_{x}\right|^{2}+\left|U_{t}\right|^{2} d x .
\end{aligned}
$$

From (2.18), (2.19), (2.20) we have

$$
\begin{aligned}
& |\underbrace{\int_{0}^{L} \alpha(x) U U_{t} d x+\frac{1}{2} \int_{0}^{L} a(x) \alpha(x)|U|^{2} d x+\int_{0}^{L} q(x) U_{x} U_{t} d x}_{:=R}| \\
& \leq|\alpha|_{\infty} \frac{1}{2} \int_{0}^{L} 2|U|^{2}+\left|U_{t}\right|^{2} d x+|q|_{\infty} \frac{1}{2} \int_{0}^{L}\left|U_{x}\right|^{2}+\left|U_{t}\right|^{2} d x
\end{aligned}
$$

Using Poincare's inequality we have

$$
\begin{aligned}
|R| & \leq|\alpha|_{\infty} \frac{1}{2} \int_{0}^{L} 2 C_{p}\left|U_{x}\right|^{2}+\left|U_{t}\right|^{2} d x+|q|_{\infty} \frac{1}{2} \int_{0}^{L}\left|U_{x}\right|^{2}+\left|U_{t}\right|^{2} d x \\
& \leq b_{2} E(t, U)
\end{aligned}
$$


where $b_{2}$ is a positive constant. From (2.21) it follows that

$$
\int_{0}^{L} \alpha(x) U U_{t} d x+\frac{1}{2} \int_{0}^{L} a(x) \alpha(x)|U|^{2} d x+\int_{0}^{L} q(x) U_{x} U_{t} d x \leq b_{2} E(t, U)
$$

Recalling the definition of $L(t, U)$ and using (2.22) we arrive at

$$
L(t, U) \leq \underbrace{\left\{N+b_{2}\right\}}_{:=k_{2}} E(t, U)
$$

on the other hand, using (2.21) we get

$$
L(t, U) \geq \underbrace{\left\{N-b_{2}\right\}}_{:=k_{1}} E(t, U)
$$

Taking $N$ such that $N>b_{2}$ our conclusion follows.

Let us define the following functional

$$
\mathcal{M}(t)=\|u(t)\|_{H^{3}(0, L)}^{2}+\left\|u_{t}(t)\right\|_{H^{2}(0, L)}^{2}+\left\|u_{t t}(t)\right\|_{H^{1}(0, L)}^{2}
$$

To obtain the global existence result we use the following hypotheses

$$
\mathcal{M}(0)=\left\|u_{0}\right\|_{H^{3}(0, L)}^{2}+\left\|u_{1}\right\|_{H^{2}(0, L)}^{2}+\left\|u_{t t}(0)\right\|_{H^{1}(0, L)}^{2}<\varepsilon^{2}
$$

for $\varepsilon>0$ small enough. Since the local solution is continuous it follows that there exists $t_{0}>0$ such that

$$
\mathcal{M}(t)<\varepsilon^{2} \quad \forall t \in\left[0, t_{0}\right]
$$

Let us define

$$
T^{*}=\sup \left\{t_{1}, \mathcal{M}(t)<d \varepsilon^{2}, t \in\left[0, t_{1}[\}\right.\right.
$$

and let us suppose that $T^{*}<\infty$. Therefeore

$$
\mathcal{M}(t)<d \varepsilon^{2} \quad \forall t \in\left[0, T^{*}[\right.
$$

Using Gagliardo Niremberg's inequality we get

$$
\left|u_{x}(x, t)\right| \leq c_{1} \varepsilon .
$$


By the mean value theorem, (2.23) and the continuity of ${ }^{\prime \prime} \sigma^{\prime \prime}$ we have

$$
\left|\sigma^{\prime}\left(u_{x}\right)-\sigma^{\prime}(0)\right| \leq c_{2} \varepsilon
$$

Using Gagliardo Niremberg's inequality and (2.23) we get

$$
\left|u_{x x}\right|<c \varepsilon,\left|u_{x t}\right|<c \varepsilon
$$

From equation (2.1) we have

$$
\begin{aligned}
& \int_{0}^{L}\left|u_{x x}\right|^{2} d x \leq 2 c_{3} \int_{0}^{L}\left|u_{t t}\right|^{2}+\left|u_{t}\right|^{2} d x \\
& \int_{0}^{L}\left|u_{x x t}\right|^{2} d x \leq 3 c_{3} \hat{c}^{2} \int_{0}^{L}\left|u_{t t t}\right|^{2}+\left|u_{t t}\right|^{2}+\left|u_{t}\right|^{2} d x
\end{aligned}
$$

where

$$
\begin{aligned}
& c_{3}:=\left\{\frac{1}{\sigma^{\prime}(0)-\varepsilon c_{2}}\right\}^{2} \\
& \hat{c}:=\left\{c \varepsilon \max _{[-1,1]}\left|\sigma^{\prime \prime}(x)\right| \frac{1}{\sigma^{\prime}(0)-\varepsilon c_{2}}+1\right\}
\end{aligned}
$$

Taking $\varepsilon>0$ such that

$$
\sigma^{\prime}(0)>\varepsilon c_{2}
$$

Let us define

$$
\begin{aligned}
& L_{1}(t)=L(t, u)+\frac{N}{2} \int_{0}^{L}\left[\sigma^{\prime}\left(u_{x}\right)-\sigma^{\prime}(0)\right]\left|u_{x}\right|^{2} d x \\
& L_{2}(t)=L\left(t, u_{t}\right)+\frac{N}{2} \int_{0}^{L}\left[\sigma^{\prime}\left(u_{x}\right)-\sigma^{\prime}(0)\right]\left|u_{x t}\right|^{2} d x \\
& L_{3}(t)=L\left(t, u_{t t}\right)+\frac{N}{2} \int_{0}^{L}\left[\sigma^{\prime}\left(u_{x}\right)-\sigma^{\prime}(0)\right]\left|u_{x t t}\right|^{2} d x \\
& S(t)=L_{1}(t)+L_{2}(t)+L_{3}(t)
\end{aligned}
$$

Lemma 2.4 With initial data (1.6) and $u$ local solution of problem (1.1) - (1.5) there exists positive constants $k_{3}$ and $k_{4}$ such that 


$$
\begin{aligned}
& k_{3} E(t, u) \leq L_{1}(t) \leq k_{4} E(t, u) \\
& k_{3} E\left(t, u_{t}\right) \leq L_{2}(t) \leq k_{4} E\left(t, u_{t}\right) \\
& k_{3} E\left(t, u_{t t}\right) \leq L_{3}(t) \leq k_{4} E\left(t, u_{t t}\right)
\end{aligned}
$$

where

$$
\begin{aligned}
& k_{3}=k_{1}-\frac{N}{2} c_{2} \frac{\varepsilon}{\sigma^{\prime}(0)} \\
& k_{4}=k_{2}+\frac{N}{2} c_{2} \frac{\varepsilon}{\sigma^{\prime}(0)}
\end{aligned}
$$

for $\varepsilon$ small enough.

Proof.- This result follows easily from relations (2.14) and (2.25).

Lemma 2.5 Under the same hypotheses of Lemma 2.4, there exists a positive constant $k$ such that

$$
\frac{d S(t)}{d t} \leq-k S(t)
$$

Moreover, there exist positive constants $k_{5}$ and $k_{6}$ such that

$$
k_{5} \mathcal{M}(t) \leq S(t) \leq k_{6} \mathcal{M}(t)
$$

\section{Proof.-}

To show (2.32) we need to estimate the following expression

$$
\int_{0}^{L} F\left\{N U_{t}+\alpha U+q U_{x}\right\} d x
$$

where $(F, U)=\left\{(f, u),\left(f_{t}, u_{t}\right),\left(f_{t t}, u_{t t}\right)\right\}$,

(i) Caso $(F, U)=(f, u)$

$$
\begin{aligned}
\int_{0}^{L} f u_{t} & =\int_{0}^{L}\left[\sigma^{\prime}\left(u_{x}\right)-\sigma^{\prime}(0)\right] u_{x x} u_{t} d x \\
& =-\int_{0}^{L}\left\{\left[\sigma^{\prime}\left(u_{x}\right)-\sigma^{\prime}(0)\right] u_{t}\right\}_{x} u_{x} d x \\
& =-\int_{0}^{L} \sigma^{\prime \prime}\left(u_{x}\right) u_{x x} u_{x} u_{t} d x-\int_{0}^{L}\left[\sigma^{\prime}\left(u_{x}\right)-\sigma^{\prime}(0)\right] u_{t x} u_{x} d x \\
& =-\int_{0}^{L} \sigma^{\prime \prime}\left(u_{x}\right) u_{x x} u_{x} u_{t} d x-\frac{1}{2} \frac{d}{d t} \int_{0}^{L}\left[\sigma^{\prime}\left(u_{x}\right)-\sigma^{\prime}(0)\right]\left|u_{x}\right|^{2} d x+\frac{1}{2} \int_{0}^{L} \sigma^{\prime \prime}\left(u_{x}\right) u_{x t}\left|u_{x}\right|^{2} d x
\end{aligned}
$$


From (2.26), $\left|\sigma^{\prime \prime}\left(u_{x}\right)\right|<M$ and Young's inequality it follows

$\leq \frac{M c \varepsilon}{2} \int_{0}^{L}\left|u_{t}\right|^{2}+\left|u_{x}\right|^{2} d x+\frac{M c \varepsilon}{2} \int_{0}^{L}\left|u_{x}\right|^{2} d x-\frac{1}{2} \frac{d}{d t} \int_{0}^{L}\left[\sigma^{\prime}\left(u_{x}\right)-\sigma^{\prime}(0)\right]\left|u_{x}\right|^{2} d x$
$\leq M c \varepsilon \max \left\{\frac{2}{\sigma^{\prime}(0)}, 1\right\} E(t, u)-\frac{1}{2} \frac{d}{d t} \int_{0}^{L}\left[\sigma^{\prime}\left(u_{x}\right)-\sigma^{\prime}(0)\right]\left|u_{x}\right|^{2} d x$

From (2.26), (2.27) and Poincare's inequality we have

$$
\int_{0}^{L} f \alpha u d x \leq \varepsilon b_{3}\left\{E(t, u)+E\left(t, u_{t}\right)\right\}
$$

where $b_{3}$ is a positive constant. From (2.25) and (2.27) we get

$$
\int_{0}^{L} f q u_{x} d x \leq \varepsilon b_{4}\left\{E(t, u)+E\left(t, u_{t}\right)\right\}
$$

where $b_{4}$ is a positive constant.

(ii) Caso $(F, U)=\left(f_{t}, u_{t}\right)$

$$
\int_{0}^{L} f_{t} u_{t t} d x=\int_{0}^{L} \sigma^{\prime \prime}\left(u_{x}\right) u_{x t} u_{x x} u_{t t} d x+\int_{0}^{L}\left[\sigma^{\prime}\left(u_{x}\right)-\sigma^{\prime}(0)\right] u_{x x t} u_{t t} d x
$$

Using (2.26) we get

$$
\int_{0}^{L} f_{t} u_{t t} d x \leq \frac{M c}{2} \varepsilon \int_{0}^{L} u_{x t}^{2}+u_{t t}^{2} d x+\underbrace{\int_{0}^{L}\left[\sigma^{\prime}\left(u_{x}\right)-\sigma^{\prime}(0)\right] u_{x x t} u_{t t} d x}_{:=I_{1}}
$$

Performing an integration by parts and using (2.26) we have

$$
I_{1} \leq \frac{M c \varepsilon}{2} \int_{0}^{L}\left|u_{t t}\right|^{2}+\left|u_{x t}\right|^{2} d x+\frac{M c \varepsilon}{2} \int_{0}^{L}\left|u_{x t}\right|^{2} d x-\frac{1}{2} \frac{d}{d t} \int_{0}^{L}\left[\sigma^{\prime}\left(u_{x}\right)-\sigma^{\prime}(0)\right]\left|u_{x t}\right|^{2} d x
$$

Sustituting of (2.38) in (2.37), we have

$$
\int_{0}^{L} f_{t} u_{t t} d x \leq M c \varepsilon \max \left\{\frac{3}{2 \sigma^{\prime}(0)}, 1\right\} E\left(t, u_{t}\right)-\frac{1}{2} \frac{d}{d t} \int_{0}^{L}\left[\sigma^{\prime}\left(u_{x}\right)-\sigma^{\prime}(0)\right]\left|u_{x t}\right|^{2} d x
$$

From (2.26) and Young's inequality we get

$$
\int_{0}^{L} f_{t} \alpha u_{t} d x \leq \frac{M c}{2} \varepsilon|\alpha|_{\infty} \int_{0}^{L}\left|u_{x t}\right|^{2}+\left|u_{t}\right|^{2} d x+\underbrace{\int_{0}^{L}\left[\sigma^{\prime}\left(u_{x}\right)-\sigma^{\prime}(0)\right] u_{x x t} \alpha(x) u_{t} d x}_{:=I_{2}}
$$


To estimate $I_{2}$ we make an integration by parts

$$
\begin{aligned}
I_{2}= & -\int_{0}^{L}\left\{\left[\sigma^{\prime}\left(u_{x}\right)-\sigma^{\prime}(0)\right] \alpha(x) u_{t}\right\}_{x} u_{x t} d x \\
= & -\int_{0}^{L} \sigma^{\prime \prime}\left(u_{x}\right) \alpha(x) u_{t} u_{x t} d x-\int_{0}^{L}\left[\sigma^{\prime}\left(u_{x}\right)-\sigma^{\prime}(0)\right] \alpha^{\prime}(x) u_{t} u_{x t} d x \\
& -\int_{0}^{L}\left[\sigma^{\prime}\left(u_{x}\right)-\sigma^{\prime}(0)\right] \alpha(x)\left|u_{x t}\right|^{2} d x
\end{aligned}
$$

Using (2.26) and (2.25) once more

$$
\begin{aligned}
I_{2} \leq & \frac{M c}{2} \varepsilon|\alpha|_{\infty} \int_{0}^{L}\left|u_{x t}\right|^{2}+\left|u_{t}\right|^{2} d x+\frac{c_{2}}{2} \varepsilon\left|\alpha^{\prime}\right|_{\infty} \int_{0}^{L}\left|u_{x t}\right|^{2}+\left|u_{t}\right|^{2} d x+ \\
& c_{2} \varepsilon|\alpha|_{\infty} \int_{0}^{L}\left|u_{x t}\right|^{2} d x
\end{aligned}
$$

Inserting (2.41) into (2.40) we conclude that

$$
\int_{0}^{L} f_{t} \alpha u_{t} d x \leq \varepsilon b_{5}\left\{E\left(t, u_{t}\right)+E(t, u)\right\}
$$

where $b_{5}$ is a positive constant. Using (2.26), (2.25) and (2.28) we have that

$$
\int_{0}^{L} f_{t} q u_{x t} d x \leq b_{6} \varepsilon\left\{E\left(t, u_{t}\right)+E\left(t, u_{t t}\right)+E(t, u)\right\}
$$

where $b_{6}>0$.

(iii) Caso $(F, U)=\left(f_{t t}, u_{t t}\right)$

$$
\begin{aligned}
\int_{0}^{L} f_{t t} u_{t t t} d x & =\int_{0}^{L} \sigma^{\prime \prime \prime}\left(u_{x}\right)\left|u_{x t}\right|^{2} u_{x x} u_{t t t} d x+\int_{0}^{L} \sigma^{\prime \prime} u_{x t t} u_{x x} u_{t t t} d x+ \\
& 2 \int_{0}^{L} \sigma^{\prime \prime}\left(u_{x}\right) u_{x t} u_{x x t} u_{t t t} d x+\int_{0}^{L}\left[\sigma^{\prime}\left(u_{x}\right)-\sigma^{\prime}(0)\right] u_{x x t} u_{t t t} d x
\end{aligned}
$$

Using (2.26), $\left|\sigma^{\prime \prime \prime}\left(u_{x}\right)\right| \leq M_{1}$ and Young's inequality

$$
\begin{array}{r}
\leq M_{1} c \frac{\varepsilon^{2}}{2} \int_{0}^{L}\left|u_{x x}\right|^{2}+\left|u_{t t t}\right|^{2} d x+M c \frac{\varepsilon}{2} \int_{0}^{L}\left|u_{x t t}\right|^{2}+\left|u_{t t t}\right|^{2} d x+ \\
M c \varepsilon \int_{0}^{L}\left|u_{x x t}\right|^{2}+\left|u_{t t t}\right|^{2} d x+\underbrace{\int_{0}^{L}\left[\sigma^{\prime}\left(u_{x}\right)-\sigma^{\prime}(0)\right] u_{x x t t} u_{t t t} d x}_{:=I_{3}}
\end{array}
$$


Integrating by parts

$$
\begin{aligned}
I_{3}= & -\int_{0}^{L}\left\{\left[\sigma^{\prime}\left(u_{x}\right)-\sigma^{\prime}(0)\right] u_{t t t}\right\}_{x} u_{x t t} d x \\
= & -\int_{0}^{L} \sigma^{\prime \prime}\left(u_{x}\right) u_{x x} u_{t t t} u_{x t t} d x-\int_{0}^{L}\left[\sigma^{\prime}\left(u_{x}\right)-\sigma^{\prime}(0)\right] u_{t t t x} u_{x t t} d x \\
= & -\int_{0}^{L} \sigma^{\prime \prime}\left(u_{x}\right) u_{x x} u_{t t t} u_{x t t} d x-\frac{1}{2} \frac{d}{d t} \int_{0}^{L}\left[\sigma^{\prime}\left(u_{x}\right)-\sigma^{\prime}(0)\right]\left|u_{x t t}\right|^{2} \\
& +\frac{1}{2} \int_{0}^{L} \sigma^{\prime \prime}\left(u_{x}\right) u_{x t}\left|u_{x t t}\right|^{2} d x
\end{aligned}
$$

Using (2.26) we get

$$
I_{3} \leq M c \frac{\varepsilon}{2} \int_{0}^{L} 2\left|u_{x t t}\right|^{2}+\left|u_{t t t}\right|^{2} d x-\frac{1}{2} \frac{d}{d t} \int_{0}^{L}\left[\sigma^{\prime}\left(u_{x}\right)-\sigma^{\prime}(0)\right]\left|u_{x t t}\right|^{2} d x
$$

Substitution of (2.45) into (2.44) yields

$$
\begin{aligned}
\int_{0}^{L} f_{t t} u_{t t t} \leq \varepsilon & \max \left\{M_{1} c \frac{\varepsilon}{2}, M c \frac{3}{2}\right\} \int_{0}^{L}\left|u_{x t t}\right|^{2}+\left|u_{x x t}\right|^{2}+\left|u_{x x}\right|^{2}+3\left|u_{t t t}\right|^{2} d x \\
& -\frac{1}{2} \frac{d}{d t} \int_{0}^{L}\left[\sigma^{\prime}\left(u_{x}\right)-\sigma^{\prime}(0)\right]\left|u_{x t t}\right|^{2} d x
\end{aligned}
$$

Using (2.27) e (2.28) we get

$$
\begin{aligned}
\int_{0}^{L} f_{t t} u_{t t t} \leq & \varepsilon b_{7}\left\{E\left(t, u_{t t}\right)+E\left(t, u_{t}\right)+E(t, u)\right\} \\
& -\frac{1}{2} \frac{d}{d t} \int_{0}^{L}\left[\sigma^{\prime}\left(u_{x}\right)-\sigma^{\prime}(0)\right]\left|u_{x t t}\right|^{2} d x
\end{aligned}
$$

where $b_{\uparrow}>0$. On the other hand, using (2.26), we have

$$
\begin{aligned}
\int_{0}^{L} f_{t t} \alpha u_{t t} & \leq \frac{M_{1} c \varepsilon}{2}|\alpha|_{\infty} \int_{0}^{L}\left|u_{x x}\right|^{2}+\left|u_{t t}\right|^{2} d x+\frac{M c \varepsilon}{2}|\alpha|_{\infty} \int_{0}^{L}\left|u_{x t t}\right|^{2}+\left|u_{t t}\right|^{2} d x \\
& +M c \varepsilon|\alpha|_{\infty} \int_{0}^{L}\left|u_{x x t}\right|^{2}+\left|u_{t t}\right|^{2} d x+\underbrace{\int_{0}^{L} \alpha(x)\left[\sigma^{\prime}\left(u_{x}\right)-\sigma^{\prime}(0)\right] u_{x x t t} u_{t t}}_{:=I_{4}}
\end{aligned}
$$


Integrating by parts

$$
\begin{aligned}
I_{4}= & -\int_{0}^{L}\left\{\alpha(x)\left[\sigma^{\prime}\left(u_{x}\right)-\sigma^{\prime}(0)\right] u_{t t}\right\}_{x} u_{x t t} d x \\
= & -\int_{0}^{L} \sigma^{\prime \prime}\left(u_{x}\right) u_{x x x} u_{t t} \alpha(x) u_{x t t} d x-\int_{0}^{L} \alpha(x)\left[\sigma^{\prime}\left(u_{x}\right)-\sigma^{\prime}(0)\right]\left|u_{t t x}\right|^{2} d x \\
& -\int_{0}^{L} \alpha(x)\left[\sigma^{\prime}\left(u_{x}\right)-\sigma^{\prime}(0)\right] u_{t t} u_{x t t} d x
\end{aligned}
$$

Using (2.26) and (2.25) we get

$$
\begin{aligned}
I_{4} \leq & \frac{M c \varepsilon}{2}|\alpha|_{\infty} \int_{0}^{L}\left|u_{x t t}\right|^{2}+\left|u_{t t}\right|^{2} d x+c_{2} \varepsilon|\alpha|_{\infty} \int_{0}^{L}\left|u_{x t t}\right|^{2} d x+ \\
& \frac{c_{2} \varepsilon}{2}\left|\alpha^{\prime}\right|_{\infty} \int_{0}^{L}\left|u_{x t t}\right|^{2}+\left|u_{t t}\right|^{2} d x
\end{aligned}
$$

Subsititution of (2.48) into (2.47) yields

$$
\int_{0}^{L} f_{t t} \alpha u_{t t} d x \leq 4 \varepsilon c_{4} \cdot \int_{0}^{L}\left|u_{x x}\right|^{2}+\left|u_{t t}\right|^{2}+\left|u_{x t t}\right|^{2}+\left|u_{x x t}\right|^{2} d x
$$

Where

$$
c_{4}:=\max \left\{\frac{M_{1} c}{2}|\alpha|_{\infty}, M c|\alpha|_{\infty}, c_{2}|\alpha|_{\infty}, \frac{c_{1}}{2}\left|\alpha^{\prime}\right|_{\infty}\right\}
$$

Using (2.27) e (2.28) we have

$$
\int_{0}^{L} f_{t t} \alpha u_{t t} d x \leq \varepsilon b_{8}\left\{E\left(t, u_{t t}\right)+E\left(t, u_{t}\right)+E(t, u)\right\}
$$

for some $b_{8}>0$.

Using (2.26) we get

$$
\begin{aligned}
\int_{0}^{L} f_{t t} q(x) u_{x t t} d x & \leq \frac{M_{1} c}{2} \varepsilon|q|_{\infty} \int_{0}^{L}\left|u_{x x}\right|^{2}+\left|u_{x t t}\right|^{2} d x+ \\
& M c \varepsilon|q|_{\infty} \int_{0}^{L}\left|u_{x t t}\right|^{2} d x+ \\
& M c \varepsilon|q|_{\infty} \int_{0}^{L}\left|u_{x x t}\right|^{2}+\left|u_{x t t}\right|^{2} d x+ \\
& \underbrace{\int_{0}^{L}\left[\sigma^{\prime}\left(u_{x}\right)-\sigma^{\prime}(0)\right] q(x) \frac{1}{2} \frac{d}{d t}\left\{\left|u_{x t t}\right|^{2}\right\} d x}_{:=I_{5}}
\end{aligned}
$$


Integrating by parts and using $q(0)=q(L)=0$ we get

$$
\begin{aligned}
I_{5} & =-\frac{1}{2} \int_{0}^{L}\left[\sigma^{\prime}\left(u_{x}\right)-\sigma^{\prime}(0)\right] q(x)_{x}\left|u_{x t t}\right|^{2} d x \\
& =-\frac{1}{2} \int_{0}^{L} \sigma^{\prime \prime}\left(u_{x}\right) u_{x x} q(x)\left|u_{x t t}\right|^{2} d x-\frac{1}{2} \int_{0}^{L}\left[\sigma^{\prime}\left(u_{x}\right)-\sigma^{\prime}(0)\right] q^{\prime}(x)\left|u_{x t t}\right|^{2} d x
\end{aligned}
$$

From (2.26) and (2.25) we get

$$
I_{5} \leq \frac{M c}{2} \varepsilon|q|_{\infty} \int_{0}^{L}\left|u_{x t t}\right|^{2} d x+\frac{c_{2}}{2} \varepsilon\left|q^{\prime}\right|_{\infty} \int_{0}^{L}\left|u_{x t t}\right|^{2} d x
$$

Substitution of (2.51) into (2.50) produces

$$
\int_{0}^{L} f_{t t} q(x) u_{x t t} d x \leq \varepsilon c_{5} \cdot \int_{0}^{L}\left\{\left|u_{x x}\right|^{2}+5\left|\dot{u}_{x t t}\right|^{2}+\left|u_{x x t}\right|^{2}\right\} d x
$$

where

$$
c_{5}:=\max \left\{\frac{M_{1} c}{2}|q|_{\infty}, M c|q|_{\infty}, \frac{c_{2}}{2}\left|q^{\prime}\right|_{\infty}\right\}
$$

Using (2.27) and (2.28) we get

$$
\begin{array}{r}
\int_{0}^{L} f_{t t} q(x) u_{x t t} d x \leq \varepsilon c_{5} \cdot \max \left\{2 c_{3}, 2 \hat{c}^{2} c_{3}, \frac{5}{\sigma^{\prime}(0)}\right\} \\
\cdot\left\{E\left(t, u_{t t}\right)+E\left(t, u_{t}\right)+E(t, u)\right\}
\end{array}
$$

Summing up (2.34), (2.35), (2.36), (2.39), (2.42), (2.43), (2.46), (2.49) and (2.52) we get

$$
\begin{aligned}
& \sum_{i=0}^{2} \int_{0}^{L} F_{i}\left\{N u_{i, t}+\alpha u_{i}+q u_{i, x}\right\} d x \leq \varepsilon \sum_{i=0}^{2} \gamma_{i} E\left(t, u_{i}\right)-\frac{N}{2} \frac{d}{d t} \int_{0}^{L}\left[\sigma^{\prime}\left(u_{x}\right)-\sigma^{\prime}(0)\right]\left|u_{x}\right|^{2} d x- \\
& \frac{N}{2} \frac{d}{d t} \int_{0}^{L}\left[\sigma^{\prime}\left(u_{x}\right)-\sigma^{\prime}(0)\right]\left|u_{t x}\right|^{2} d x-\frac{N}{2} \frac{d}{d t} \int_{0}^{L}\left[\sigma^{\prime}\left(u_{x}\right)-\sigma^{\prime}(0)\right]\left|u_{t t x}\right|^{2} d x
\end{aligned}
$$

with the following notations 


$$
\begin{array}{ll}
F_{0}=f, & , u_{0}=u \\
F_{1}=f_{t}, & u_{1}=u_{t} \\
F_{2}=f_{t t} & , u_{2}=u_{t t}
\end{array}
$$

where $\left\{\gamma_{i}\right\}_{i=0}^{2}$ are positive constants depending on $\varepsilon, c_{p}, \sigma^{\prime}(0)$, of the $L^{\infty}$ norm of the functions $\alpha, q, \sigma^{\prime}, q^{\prime}, \sigma^{\prime \prime}\left(u_{x}\right)$ and $\sigma^{\prime \prime \prime}\left(u_{x}\right)$.

Using Lemma 2.3 and (2.53) we have

$$
\frac{d S(t)}{d t} \leq-\sum_{i=0}^{2}\left(\hat{\delta}-\varepsilon \gamma_{i}\right) E\left(t, u_{i}\right)
$$

where $\left\{\hat{\delta}-\varepsilon \gamma_{i}\right\}_{i=0}^{2}$ is positive for $\varepsilon$ small enough. From (2.54), (2.29), (2.30), (2.31) we have

$$
\begin{aligned}
\frac{d S(t)}{d t} & \leq-\frac{1}{k_{4}}\left\{\left(\hat{\delta}-\varepsilon \gamma_{0}\right) L_{1}(t)+\left(\hat{\delta}-\varepsilon \gamma_{1}\right) L_{2}(t)+\left(\hat{\delta}-\varepsilon \gamma_{2}\right) L_{3}(t)\right\} \\
& \leq-k S(t)
\end{aligned}
$$

where

$$
k:=\frac{1}{k_{4}} \min _{i=0,1,2}\left\{\hat{\delta}-\varepsilon \gamma_{i}\right\}
$$

From where (2.32) follows. To show (2.33) we need the following estimates

$$
\begin{aligned}
& \int_{0}^{L}\left|u_{t t t}\right|^{2} d x \leq k_{7} \int_{0}^{L}\left|u_{x x t}\right|^{2}+\left|u_{t}\right|^{2}+\left|u_{x x}\right|^{2} d x \\
& \int_{0}^{L}\left|u_{x x x}\right|^{2} d x \leq k_{8} \int_{0}^{L}\left|u_{x t}\right|^{2}+\left|u_{x t t}\right|^{2}+\left|u_{x x}\right|^{2}+\left|u_{t}\right|^{2} d x
\end{aligned}
$$

where $k_{7}$ and $k_{8}$ positive constants. This inequalities follows differentiating relation (2.1) with respect to $x$ and $t$, and using the same above reasoning. From the definition of $S$ and relations (2.29), (2.30), (2.31) we get

$$
S(t) \leq k_{4}\left\{E(t, u)+E\left(t, u_{t}\right)+E\left(t, u_{t t}\right)\right\}
$$

Then, from (2.55) we can conclude that

$$
S(t) \leq k_{6} \mathcal{M}(t)
$$


for some $k_{6}>0$. On the other hand, recalling the definition of $S$ and keeping in mind relations (2.29), (2.30), (2.31) we have

$$
S(t) \geq k_{3}\left\{E(t, u)+E\left(t, u_{t}\right)+E\left(t, u_{t t}\right)\right\}
$$

Using (2.27), (2.28), (2.56) and Poincare's inequality we arrive at

$$
S(t) \geq k_{5} \mathcal{M}(t)
$$

for $k_{5}>0$. Therefores (2.33) follows.

Integrating (2.32) from 0 to $t$ and letting $t \rightarrow T^{*}$ we get

$$
S\left(T^{*}\right)<S(0)
$$

Applying (2.33) is follows that

$$
k_{5} \mathcal{M}\left(T^{*}\right) \leq S\left(T^{*}\right)<S(0) \leq k_{6} \mathcal{M}(0)
$$

that is

$$
\mathcal{M}\left(T^{*}\right) \leq \frac{k_{6}}{k_{5}} \mathcal{M}(0)<\frac{k_{6}}{k_{5}} \varepsilon^{2}
$$

Taking $d=\frac{k_{6}}{k_{5}}$. There exists $T_{1}>T^{*}$ such that $\mathcal{M}(t)<d \varepsilon^{2}$ for any $t$ on $\left[T^{*}, T_{1}\right)$ which implies that there exists $T_{1}>T^{*}$ such that $\mathcal{M}(t)<d \varepsilon^{2}$ for any $t$ in the interval $\left[0, T_{1}\right)$, which is contradictory to the maximality of $T^{*}$. Therefore, we conclude that $T^{*}=\infty$ which implies that the solution is global in $t$. From (2.32) we get the exponential decay of $S$. Using (2.33) we get the exponential decay of $\mathcal{M}$. The proof is now complete. 


\section{REFERENCES}

[ 1 ] Adams, R. A. Sobolev Spaces. Academic Press, New York (1975).

[ 2 ] Bardos, C. Lebeau, G. Rauch, J. Un exemple d'utilisation des notions de propagation pour le contrôle et la estabilisation des problèmes hyperboliques. The procedings of the workshop on non linear hyperbolic equations in applied sciences (edited by N. Bellono), Rendiconti del Seminario Matematico, Universita'e Politécnico Torino, 11 - 31 (Fascicolo Speciale 1988).

[3] Dafermos, C. M. Asymptotic behavior of solutions of evolution equations, in «Nonlinear Evolution Equations», M. G. Grandall Ed., Academic Press, New York, p. 103 - 203 (1978).

[4] Haraux, A. Stabilization of trajectories for some weakly damped hyperbolic equations. J. Differential Equations 59, 145 - 154 (1985).

[ 5 ] Martinez, P. Decay of solutions of the wave equation with a local highly degenerate dissipation. Asymptotic Analysis 19, 1 - 17 (1999).

[6] Renardy, M. Hrusa, W. J. and Nohel, J. A. Mathematical Problems in Viscoelasticity. Pitman Monographs and Surveys in Pure and Applied Mathematics 35, John Wiley \& Sons, Inc., New York (1987).

[ 7 ] Rivera, J. M. and Rioco, K. B. Existence and exponential decay in nonlinear thermoleasticity. Nonlinear Analysis, Theory, Methods \& Applications, 31(1\&2), 149 - 162 (1998).

[8] Zuazua, E. Exponential decay for the semi-linear wave equation with locally distributed damping. Comm. P. D. E. 15, 205 - 235 (1990). 